\title{
Utility of Scintigraphy in Assessment of Noninfectious Complications of Peritoneal Dialysis
}

\author{
Gagandeep Choudhary ${ }^{1}$, Padma P. Manapragada ${ }^{1}$, Eric Wallace ${ }^{2}$, and Pradeep Bhambhvani ${ }^{1}$ \\ ${ }^{I}$ Division of Molecular Imaging and Therapeutics, Department of Radiology, University of Alabama at Birmingham, Birmingham, \\ Alabama; and ${ }^{2}$ Division of Nephrology, Department of Medicine, University of Alabama at Birmingham, Birmingham, Alabama
}

\begin{abstract}
Dialysis is an artificial process to remove waste products and excess water from the body in patients with kidney failure. Two main types of dialysis are available. Hemodialysis, which uses an artificial filtration apparatus, is usually done at specialized centers but can be done in a patient's home. Peritoneal dialysis functions by placing dialysis fluid, also called dialysate, into the peritoneal cavity, allowing for solute to be removed from the peritoneal capillaries through diffusion across a chemical gradient into the dialysate and removal of water through an osmotic gradient created by hypertonic dextrose. Peritoneal dialysis can be either automated, which is done with the help of a machine called a cycler, or continuous ambulatory, which is a process involving multiple exchanges a day and is performed using only gravity to infuse and drain the solution from the peritoneal cavity. For many reasons, the number of people using home dialysis has recently started to rise, with the largest increase in the United States occurring after the implementation of the prospective bundled payment system for end-stage renal disease. With the increased use of home dialysis, potential complications will increase as well. It is imperative that our health-care system be poised not only to increase the number of home dialysis patients but also to diagnose and manage any complications. Nuclear imaging is a commonly available modality to detect various complications related to peritoneal dialysis. In this review article, we discuss the role of peritoneal scintigraphy in detecting some noninfectious peritoneal dialysis complications, with emphasis on scintigraphy technique; imaging time points; the role of planar, SPECT, and SPECT/CT imaging; and the clinical indications, with illustrative case examples.
\end{abstract}

Key Words: peritoneal scintigraphy; peritoneal dialysis; continuous ambulatory peritoneal dialysis; dialysis complications

J Nucl Med Technol 2019; 47:163-168

DOI: 10.2967/jnmt.118.223156

\section{$\mathbf{P}$} eritoneal dialysis is an effective and widely used renal replacement therapy in patients with end-stage kidney disease. Around 197,000 patients are on peritoneal dialysis

\footnotetext{
Received Nov. 7, 2018; revision accepted Nov. 26, 2018.

For correspondence or reprints contact: Gagandeep Choudhary, Division of Molecular Imaging and Therapeutics (MIT), Department of Radiology, University of Alabama at Birmingham, 619 19th St. S., Birmingham, AL 35249.

E-mail: gchoudhary@uabmc.edu

Published online Jan. 29, 2019.

COPYRIGHT @ 2019 by the Society of Nuclear Medicine and Molecular Imaging.
}

worldwide, representing $11 \%$ of dialysis patients (2). Peritoneal dialysis can be performed using a cycler at night, which automates the process while the patient sleeps, termed automated peritoneal dialysis, or using manual exchanges in a continuous fashion, termed continuous ambulatory peritoneal dialysis. Peritoneal dialysis is generally performed 7 days a week. However, incremental peritoneal dialysis can at times allow for less frequent therapy in patients with significant residual kidney function.

The continuous ambulatory technique was first described in the 1950s (3). Dialysate is injected into the peritoneal cavity via a catheter entering through the anterior abdominal wall and piercing the parietal peritoneum with its tip positioned in the pelvis. The peritoneum, with its fenestrated capillary bed, functions as a semipermeable membrane for exchange of electrolytes, glucose, urea, albumin, and other small molecules from the blood. Typically, $2-2.5 \mathrm{~L}$ of dialysate is infused via a peritoneal dialysis catheter into the peritoneal cavity (4), which can also increase the intraabdominal pressure and contribute to certain complications of this treatment. The dialysate distributes throughout the peritoneal cavity and uses the available peritoneal membrane surface area, primarily the parietal peritoneum, to enable solute exchange and fluid removal. The pelvis holds the greatest volume of dialysate. The chemical gradient provided by the dialysate solution enables solute exchange, whereas hypertonic dextrose in the dialysate solution provides an osmotic gradient by which fluid is removed from the patient.

Peritoneal dialysis has an advantage over hemodialysis, as it allows patients greater freedom to perform daily activities. Other advantages include speed and ease of use, lower cost, and absence of the need for a highly skilled operator and anticoagulation. Furthermore, data suggest an improvement in transplant outcomes in patients who have been using peritoneal dialysis versus hemodialysis, as well as a positive effect on preservation of residual kidney function. Data also suggest that there is less myocardial stunning in peritoneal dialysis than in hemodialysis, and as such, it may be the modality of choice in patients with cardiovascular comorbidities. Furthermore, it provides an alternative means of dialysis for patients in whom vascular access is challenging $(5,6)$. Despite its advantages, peritoneal dialysis is not without potential complications, both infectious and noninfectious (7). 


\section{INFECTIOUS COMPLICATIONS}

Infectious complications of peritoneal dialysis include catheter exit site infection, tunnel infection, and peritonitis. Peritonitis is a major complication of peritoneal dialysis and remains the primary reason that patients switch from peritoneal dialysis to hemodialysis, otherwise known as technique failure. Treatment for peritoneal dialysisassociated peritonitis consists of antimicrobial therapy, delivered most commonly intraperitoneally, and in severe cases catheter removal (8). Exit site infections are usually curable with antimicrobial treatment but can progress to tunnel infections. Tunnel infection that fails to resolve may require removal of the peritoneal dialysis catheter and surgical implantation of a new catheter at a different site.

\section{NONINFECTIOUS COMPLICATIONS}

Some of the noninfectious complications include catheter dysfunction or poor drainage, dialysate leakage either subcutaneously or as a peritoneal-pleural fistula, peritoneal herniation, weakening of the abdominal wall, and sclerosing encapsulating peritonitis. Infusion of dialysate into the peritoneal cavity leads to increased intraabdominal pressure, which can result in herniation of the peritoneum or leakage of dialysate (7). The functioning of peritoneal dialysis can also be compromised by peritoneal adhesions and loculations that restrict dialysate distribution (4). Peritoneal scintigraphy can play a key role in identifying these noninfectious complications.

Peritoneal cavity imaging can be done with radiography, nuclear scintigraphy, ultrasound, MRI, and CT with intraperitoneal iodinated contrast agent.

Plain-film abdominal radiography can help to detect bowel obstruction, constipation, migration of peritoneal dialysis catheters, pneumoperitoneum, and peritoneal calcifications.

CT peritoneography has the disadvantage of exposure to ionizing radiation and involves a peritoneal dialysis technician who instills $100 \mathrm{~mL}$ of iodinated contrast agent in a sterile fashion into a 2-L bag of dialysate. This must then be infused into the patient 30 min before CT imaging (9).

MR peritoneography is constrained by higher costs and limited availability. In this case, the dialysate itself serves as the contrast agent, as MRI can detect water signal. As such, no added contrast agent is needed. However, protocols with gadolinium contrast mixed with dialysate have been described. Dialysate is infused into the patient, and MRI is done $30 \mathrm{~min}$ later $(7,10,11)$.

Ultrasound does not involve ionizing radiation and can identify complications such as abdominal wall or intraabdominal collections, peritoneal thickening, peritoneal calcification, or thickened and dilated small-bowel loops but has limitations due to operator dependence and lack of sensitivity, compared with CT or MRI, to rule out conditions such as focal peritoneal thickening seen in early sclerosing peritonitis $(4,12)$.
Peritoneal scintigraphy has several advantages. It is easy to perform, can be done in most hospitals with nuclear medicine facilities, produces less radiation exposure, and has a minimal risk of allergic reaction. Additional delayed imaging is also possible if needed, without added radiation exposure. It has high sensitivity in detecting relatively small volumes of leakage, with excellent diagnostic accuracy $(13)$.

\section{PERITONEAL SCINTIGRAPHY IMAGING TECHNIQUE}

There is no established standardized imaging protocol for peritoneal scintigraphy. Outlined below is the technique used in our facility in collaboration with the dialysis technician.

The study is initiated by draining any residual dialysis fluid from the abdominal cavity. After complete drainage, the patient is positioned supine under the $\gamma$-camera to allow a full view of the abdomen and lung bases. Imaging is preferably performed using a dual-head $\gamma$-camera with a low-energy high-resolution parallel-hole collimator. The photopeak is set at $140 \mathrm{keV}$, with a $20 \%$ window. The radiopharmaceutical is mixed with the peritoneal dialysis solution, warmed to body temperature, and administered rapidly into the peritoneal cavity through the abdominal catheter with simultaneous anterior dynamic imaging during the instillation phase over $15 \mathrm{~min}$ ( $1 \mathrm{~min} /$ frame $). \mathrm{Ab}$ dominal burning or pain can be caused from an overheated solution, and abdominal cramping or hypothermia may result if the peritoneal dialysis solution is administered cold. The volume and tonicity of instilled fluid varies with every patient (average volume, $2 \mathrm{~L}$ ). After the completion of infusion, static anterior, posterior, and, as needed, lateral images of the abdomen, including the lung bases, are acquired ( 5 min/acquisition). In cases of suspected pleural-peritoneal fistula, static anterior and posterior images of the chest are also acquired. The patient is then advised the walk for 10-15 min. Nonambulatory patients are asked to roll from side to side to promote mixing and even distribution of peritoneal fluid $(14,15)$. Postambulatory static images are taken in anterior, posterior, and, as needed, lateral projections similarly to the postinfusion images. The nuclear physician reviews these images, and if they are deemed adequate, the study is completed by removal of peritoneal dialysis fluid and acquisition of static postdrainage images. With today's wider availability of SPECT/CT cameras, regional SPECT/CT can also be performed for better localization of the abnormality seen on static planar images. However, if initial images are not conclusive and a leak is strongly suspected, additional delayed images can be performed at the 4-h and 24-h time points with or without additional SPECT/CT imaging (16,17). Delayed images can also help demonstrate the full extent of the leakage. The preferred radiopharmaceutical is nonabsorbable, with large particles, to prevent passage across the normal peritoneum. Sterile ${ }^{99 \mathrm{~m} T c-s u l f u r}$ colloid is the most commonly 
used tracer, with a usual dose of 37-185 MBq (1-5 mCi). ${ }^{99 \mathrm{~m}} \mathrm{Tc}$-diethylenetriaminepentaacetic acid or $99 \mathrm{~m}$ Tc-macroaggregated albumin can also be used instead of ${ }^{99 \mathrm{~m}} \mathrm{Tc}$-sulfur colloid (18).

Normal scan findings are a free flow of dialysate fluid throughout the peritoneal cavity, outlining the intraperitoneal recesses. There should be no dialysate tracking into the anterior abdominal wall, pericatheter space, pleural space, inguinal canal, or genital area, with limited residual abdominopelvic tracer activity after fluid drainage (Fig. 1) (15).

The dialysates are commercially available in different formulations with varying osmolality, dextrose strength, and calcium concentration. Dianeal PD-2 (Baxter) with 2.5\% dextrose is used at our institution (19).

\section{ROLE OF SPECT AND SPECT/CT}

SPECT and SPECT/CT provide added anatomic information that increases the sensitivity and specificity of planar scintigraphic findings. In addition to offering improved anatomic localization, SPECT/CT offers the opportunity to add diagnostic information derived from CT imaging. CT coregistration results in higher specificity and sensitivity of scintigraphic findings and markedly reduces the number of indeterminate findings (20). The low-dose CT in SPECT/CT imaging is acquired for anatomic reference and attenuation correction, with relatively less radiation dose to the patient than from a routine diagnostic CT scan (21).

SPECT/CT is especially helpful in the case of small leaks that may not be evident on planar scintigraphy because of its limited resolution $(20,22,23)$.

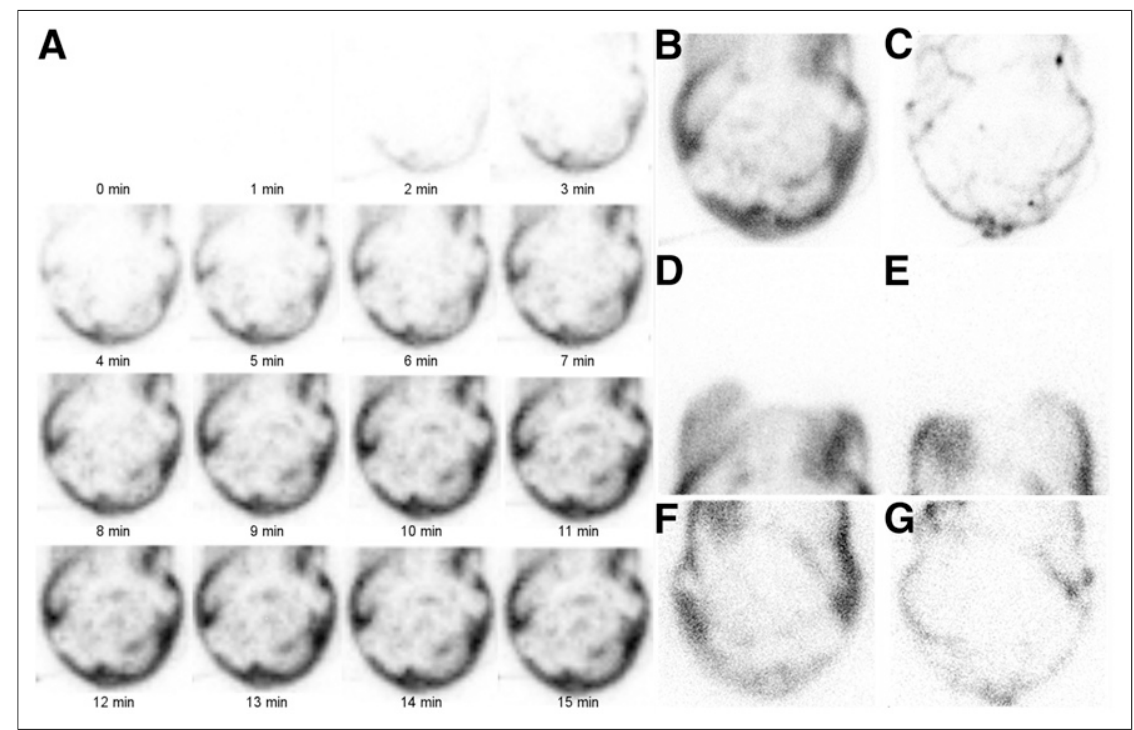

FIGURE 1. Peritoneal scintigraphy study showing normal results. (A) Fifteen-minute dynamic infusion images of abdomen demonstrate progressive accumulation of tracer into peritoneal cavity, with uniform distribution. (B-E) Postinfusion anterior and posterior static images of abdomen ( $B$ and $C$ ) and chest ( $D$ and $E$ ) demonstrate no abnormal tracer collection. ( $F$ and $G$ ) Postdrainage images also demonstrate no abnormal tracer retention.

\section{DISCUSSION}

The dialysate infusion into the peritoneal cavity leads to increased intraabdominal pressure, which can result in leakage because of tear or rupture of the peritoneal membrane. Similarly, increased pressure can also cause hernias, leading to poor drainage and fluid retention, hence compromising the functioning of continuous ambulatory peritoneal dialysis (7). Hernia formation is a frequent complication and is seen in $2.7 \%-25 \%$ of patients on this type of dialysis. Predisposing factors include prior abdominal surgery, chronic coughing and straining, and systemic conditions such as uremia, obesity, transperitoneal protein loss, and anemia, which may also lead to elevated stress on the abdominal wall and diaphragm (13). The most common indications for peritoneal scintigraphy is to check for dialysate leakage around the catheter insertion site (pericatheter leak) and into the abdominal wall or pleural cavity, as well as to check for inguinal hernia and loculated fluid collections within the abdominal cavity (15).

Leaks can be classified as an early one occurring within 30 days of catheter insertion or a late one occurring after 30 days. Most early leaks are successfully treated with temporary discontinuation of peritoneal dialysis and transferring to hemodialysis for a few days to a few weeks. Late leaks are difficult to treat, with most requiring surgical closure $(24,25)$. Peritoneal dialysis-related hydrothorax can be treated with therapeutic thoracentesis, surgical repair of the defect through video-assisted thoracic surgery, pleurodesis, or switching to hemodialysis. Leakage at the catheter insertion site may require catheter removal and reinsertion at a different site. For inguinal, scrotal, and abdominal wall hernias, surgical correction is the best option (18).

\section{Leak into Pleural Cavity}

Hydrothorax from a dialysate leak into the pleural cavity is a rare but potentially life-threatening complication of peritoneal dialysis, with an incidence of $2 \%-6 \%(18,26)$. It can occur as soon as after the first few dialysate exchanges or can be delayed for several years (7). The etiology of hydrothorax in peritoneal dialysis patients is uncertain. During continuous ambulatory peritoneal dialysis, there is continuous abdominal distention and increased intraabdominal pressure from the large volume of iatrogenic ascites, whereas the pleural cavity has negative pressure. This results in a large pressure gradient between the peritoneal and pleural cavities, which can cause a flow of fluid from the peritoneal cavity into the pleural space through the diaphragmatic foramina, anatomic defects, or 
subdiaphragmatic lymphatics. More serious complication due to a 1-way valve mechanism of the diaphragmatic defect can result in tension hydrothorax. Hydrothorax is much more common on the right side (4:1) and has a female preponderance $(18,26)$. Peritoneal scintigraphy is useful in evaluation of pleural-peritoneal leaks (Fig. 2).

\section{Inguinal or Genital Leak}

In patients on continuous ambulatory peritoneal dialysis, groin or genital swelling is usually caused by an underlying hernia due to a patent processus vaginalis. The processus vaginalis is an outpouching of parietal peritoneum, which passes through the abdominal wall toward the scrotum in male patients and the labia majora in female patients. In most individuals, the processus vaginalis closes at any point from a few weeks before birth to the first year after birth. The incidence of persistent processus vaginalis in the general adult population is $15 \%-37 \%$ (27). It is much more common in men and on the right, because of the later descent of the right testis than of the left (Fig. 3) (24).

\section{Leak into Abdominal Wall}

Abdominal hernia can develop in approximately $25 \%$ of continuous ambulatory peritoneal dialysis patients. It is more common in patients with polycystic kidney disease, a history of previous abdominal surgeries, multiparity, obesity, and steroid use $(7,14)$. Most of these hernias occur at the umbilicus, near the catheter insertion site, or in inguinal

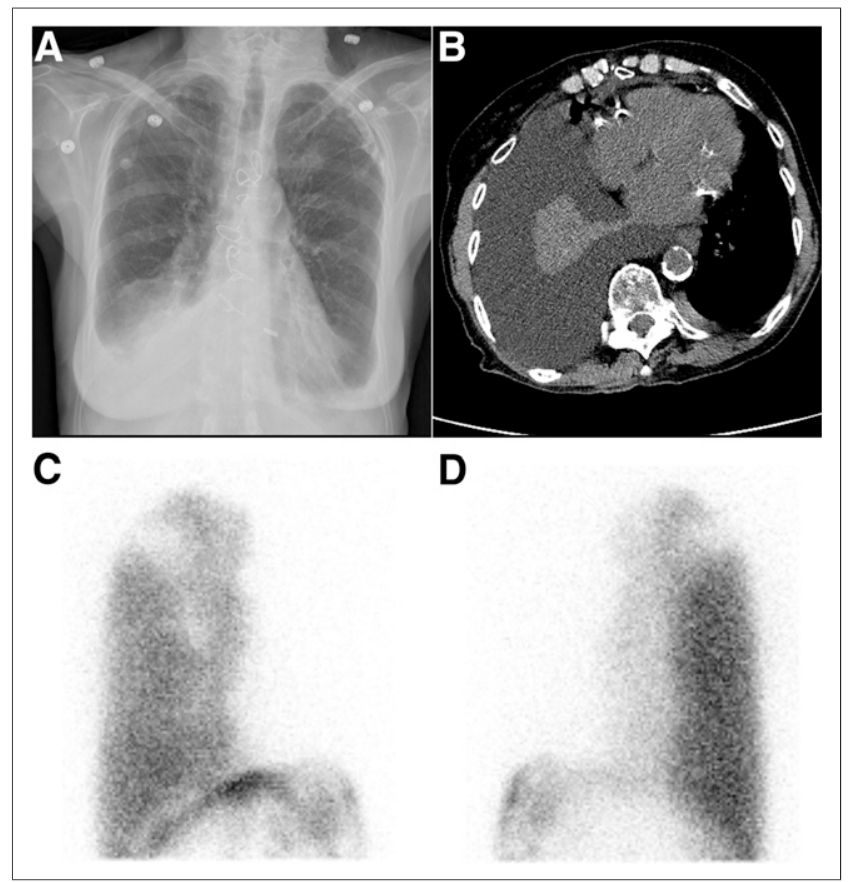

FIGURE 2. A 46-y-old woman on peritoneal dialysis who presented with acute shortness of breath and chest pain. (A and $B$ ) Chest radiograph $(A)$ and axial CT scan (B) show moderate right pleural effusion. ( $C$ and $D$ ) Anterior and posterior postinfusion static images of chest demonstrate abnormal accumulation of tracer in right pleural cavity, confirming pleural-peritoneal leak.

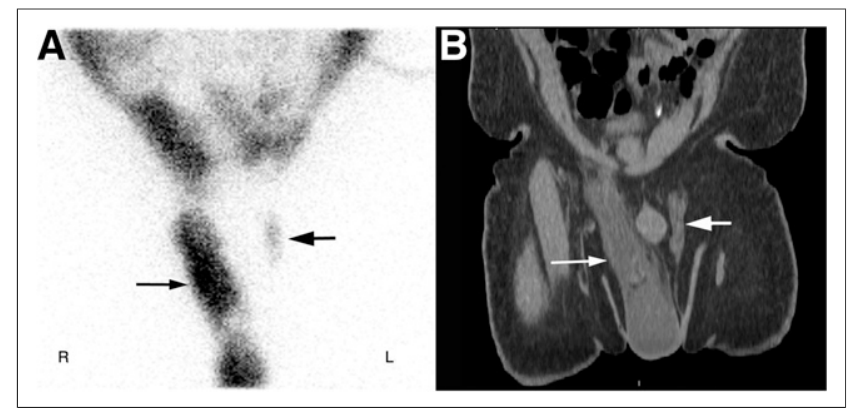

FIGURE 3. A 60-y-old man with end-stage renal disease on peritoneal dialysis who presented with right scrotal swelling. (A) Postinfusion anterior static images of pelvis show abnormal bilateral accumulation of tracer (right greater than left, arrows) in the patent processus vaginalis and inguinoscrotal regions. (B) On coronal CT image, fluid (arrows) is seen in both patent processus vaginalis and right scrotum.

regions. Dialysate leakage into an abdominal wall hernia can also extend to the scrotum along the fascia of Scarpa of the lower anterior abdominal wall (Figs. 4 and 5) (7).

\section{Loculated Collection}

Encapsulated peritoneal sclerosis is an uncommon but major threat in patients receiving continuous ambulatory peritoneal dialysis and has a high rate of mortality (24\%60\%) (28). There are peritoneal adhesions with fibrotic thickening, which can cause encapsulation of small-bowel loops. In longstanding cases there is thickening and calcification of the bowel wall and peritoneum, loculated fluid

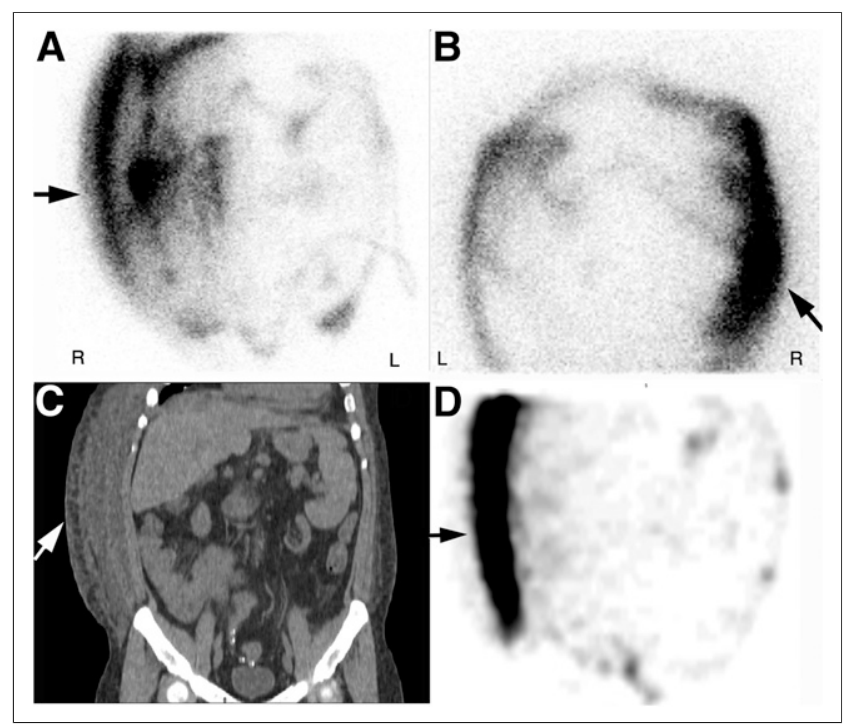

FIGURE 4. A 38-y-old man on peritoneal dialysis who was admitted to hospital with progressive right-sided abdominal swelling and shortness of breath. (A and B) Postinfusion anterior and posterior static images of abdomen show abnormal accumulation of tracer (arrows) in right lateral abdominal wall. (C and D) Subsequent CT and SPECT coronal images also demonstrate abnormal fluid and tracer collection (arrows) in right lateral abdominal wall, confirming abdominal wall leak. 


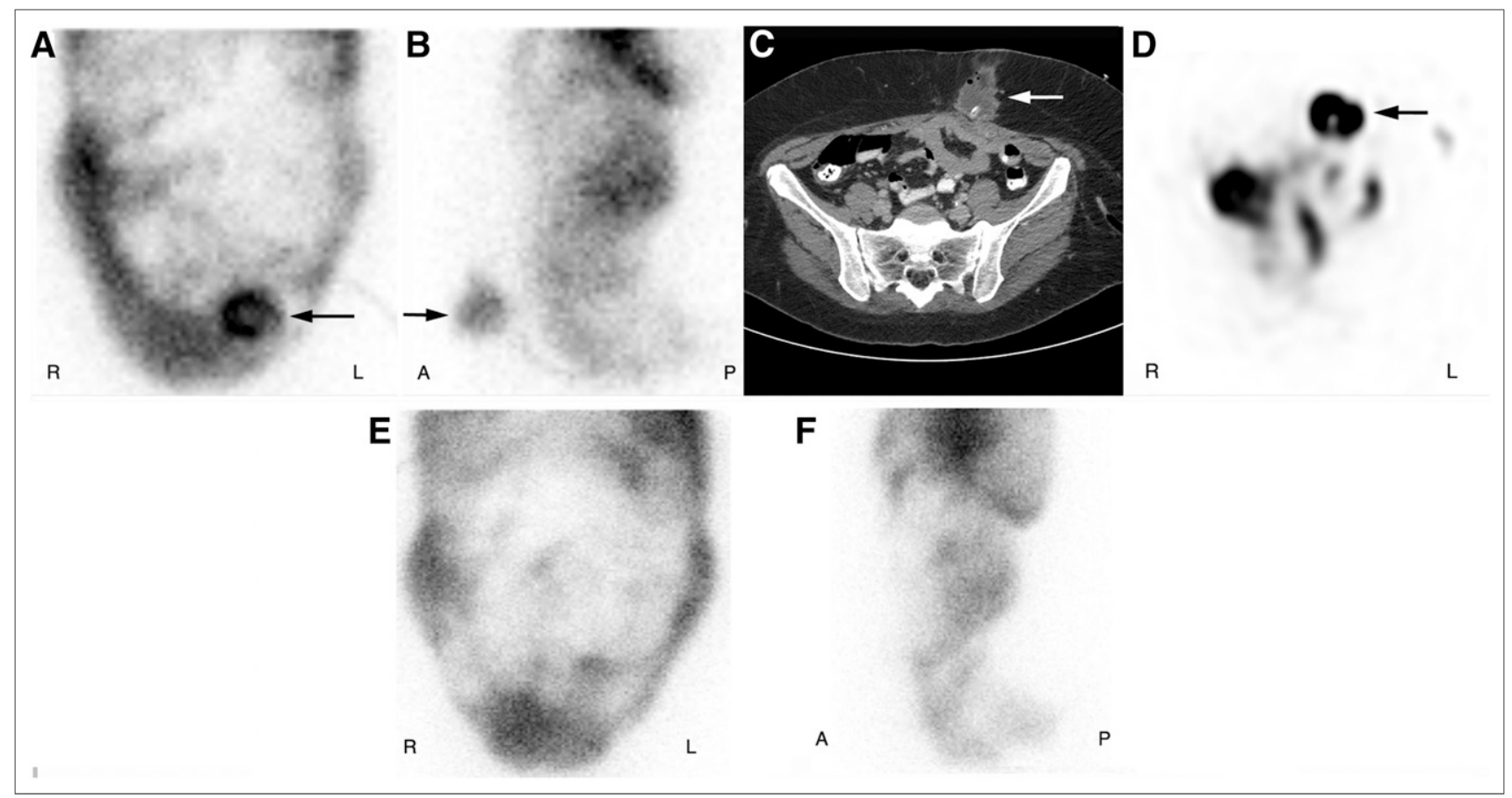

FIGURE 5. A 38-y-old man on peritoneal dialysis who presented with mild abdominal discomfort and swelling in left lower abdominal wall. (A and B) Postinfusion planar anterior and lateral images of abdomen show focal abnormal accumulation of tracer (arrows) in left lower anterior abdominal wall. (C and D) Subsequent CT and SPECT axial images demonstrate abnormal fluid and tracer accumulation (arrows) at insertion site of abdominal wall catheter, confirming pericatheter leak. Patient was treated with reinsertion of peritoneal dialysis catheter. ( $E$ and $F$ ) Follow-up postinfusion planar anterior and lateral images demonstrate normal tracer distribution in peritoneal cavity, with resolution of pericatheter leak.

collections, membranes, and partial or complete small-bowel obstruction (7). On peritoneal scintigraphy there is a nonuniform distribution with dialysate fluid confined to the parts of the abdomen with loculations. Postdrainage images show incomplete drainage of dialysate with trapping within the loculations (29).

\section{Leak into Pericardial Cavity}

Leakage of dialysate fluid into the pericardial cavity is extremely rare, with only a few cases reported in the literature (30-32). The patient can present with shortness of breath on exertion, orthopnea, and sometime life-threatening cardiac tamponade (31). It is important to differentiate this condition from the more common uremic pericarditis. Diagnosis is usually made on radiography, echocardiography, and cross-sectional imaging. However, nuclear scintigraphy may be required to confirm the peritoneal-pericardial communication. On planar scintigraphy, horseshoe-shaped pericardial effusion can be seen (32). SPECT/CT will demonstrate tracer activity in the pericardial cavity (33).

\section{CONCLUSION}

Continuous ambulatory peritoneal dialysis can be adversely affected by noninfectious structural complications such as peritoneal leaks (into the pleural cavity, abdominal wall, or inguinoscrotal region) and loculations. Confirmation and accurate localization of the underlying structural abnormality are necessary to appropriately manage these complications.
Peritoneal scintigraphy has several advantages, including being easy to perform, causing minimal radiation exposure to the patient, and having minimal risk of allergic reaction. Multipletime-point imaging, including early/infusion, postinfusion, postambulatory, postdrainage, and delayed images, can be easily obtained without additional radiation exposure. SPECT/ CT has high sensitivity in detecting relatively small volumes of leakage with excellent diagnostic accuracy. Peritoneal scintigraphy has proven to be useful in the diagnosis of complications such as anterior abdominal, pleural-peritoneal, inguinal, and genital leaks and loculations. Recognition of the various appearances of dialysate leaks on peritoneal scintigraphy is important to accurately diagnose these complications.

\section{DISCLOSURE}

No potential conflict of interest relevant to this article was reported.

\section{REFERENCES}

1. Chapter 9: Healthcare Expenditures for Persons with ESRD. U.S. Renal Data System website. https://www.usrds.org/2017/download/v2_c09_MedExp_17.pdf. Published 2017. Assessed February 19, 2019.

2. Jain AK, Blake P, Cordy P, Garg AX. Global trends in rates of peritoneal dialysis. J Am Soc Nephrol. 2012;23:533-544.

3. Maxwell MH, Rockney RE, Kleeman CR, Twiss MR. Peritoneal dialysis. 1. Technique and applications. J Am Med Assoc. 1959;170:917-924.

4. Goldstein M, Carrillo M, Ghai S. Continuous ambulatory peritoneal dialysis: a guide to imaging appearances and complications. Insights Imaging. 2013; 4:85-92. 
5. Cochran ST, Do HM, Ronaghi A, Nissenson AR, Kadell BM. Complications of peritoneal dialysis: evaluation with CT peritoneography. Radiographics. 1997;17: $869-878$.

6. Hollett MD, Marn CS, Ellis JH, Francis IR, Swartz RD. Complications of continuous ambulatory peritoneal dialysis: evaluation with CT peritoneography. AJR. 1992;159:983-989.

7. Stuart S, Booth TC, Cash CJ, et al. Complications of continuous ambulatory peritoneal dialysis. Radiographics. 2009;29:441-460.

8. Quantrill SJ, Woodhead MA, Bell CE, Hutchison AJ, Gokal R. Peritoneal tuberculosis in patients receiving continuous ambulatory peritoneal dialysis. Nephrol Dial Transplant. 2001;16:1024-1027.

9. Xu T, Xie J, Wang W, Ren H, Chen N. Peritoneal-pleural leaks demonstrated by CT peritoneography. Case Rep Nephrol Dial. 2015;5:135-139.

10. Szeto CC, Chow KM. Pathogenesis and management of hydrothorax complicating peritoneal dialysis. Curr Opin Pulm Med. 2004;10:315-319.

11. Prischl FC, Muhr T, Seiringer EM, et al. Magnetic resonance imaging of the peritoneal cavity among peritoneal dialysis patients, using the dialysate as "contrast medium." J Am Soc Nephrol. 2002;13:197-203.

12. Chow KM, Szeto CC, Li PK. Management options for hydrothorax complicating peritoneal dialysis. Semin Dial. 2003;16:389-394.

13. Bhattacharya A, Mittal BR. Peritoneo-scrotal communication: demonstration by 99mtechnetium sulphur colloid scintigraphy. Australas Radiol. 2005;49:335-337.

14. Tokmak H, Mudun A, Turkmen C, Sanli Y, Cantez S, Bozfakioglu S. The role of peritoneal scintigraphy in the detection of continuous ambulatory peritoneal dialysis complications. Ren Fail. 2006;28:709-713.

15. Juergensen PH, Rizvi H, Caride VJ, Kliger AS, Finkelstein FO. Value of scintigraphy in chronic peritoneal dialysis patients. Kidney Int. 1999;55:1111-1119.

16. Covington MF, Choudhary G, Avery RJ, Krupinski EA, Kuo PH. Optimal time points for scintigraphic imaging of pleuroperitoneal shunts. Clin Nucl Med. 2016; 41:766-768.

17. Covington MF, Choudhary G, Avery RJ, Kuo PH. Pitfalls in the performance and interpretation of scintigraphic imaging for pleuroperitoneal shunt. Clin Nucl Med. 2016;41:858-861.

18. Wu P-S, Lee B-F, Chiu N-T, Yao W-J, Huang J-J, Wang M-C. Peritoneal scintigraphy for the assessment of dialysate leakage in patients on continuous ambulatory peritoneal dialysis. Ann Nucl Med Sci. 2001;14:11-18.
19. Dianeal (dextrose) peritoneal dialysis solution [package insert]. Baxter website. http://baxterpi.com/pi-pdf/Dianeal_PI.pdf. Revised March 2018. Assessed February 19, 2019.

20. Farid K, Guyot M, Jeandot R, Durieux M, Allard M, Fernandez P. SPECT-CT improves detection of small peritoneal fistula. Clin Nucl Med. 2009;34:634-635.

21. Buck AK, Nekolla S, Ziegler S, et al. SPECT/CT. J Nucl Med. 2008;49:13051319.

22. Chen YC, Su YC, Chiu JS, Wei CK, Wang YF. Peritoneo-scrotal shunting diagnosed by Tc-99m DTPA SPECT/CT imaging. Kidney Int. 2010;78:523.

23. Tun KN, Tulchinsky M. Pericatheter leak in a peritoneal dialysis patient: SPECT/ CT diagnosis. Clin Nucl Med. 2012;37:625-628.

24. Leblanc M, Ouimet D, Pichette V. Dialysate leaks in peritoneal dialysis. Semin Dial. 2001;14:50-54.

25. Tzamaloukas AH, Gibel LJ, Eisenberg B, et al. Early and late peritoneal dialysate leaks in patients on CAPD. Adv Perit Dial. 1990;6:64-71.

26. Pankaj P, Pathak V, Sen IB, Verma R, Bhalla AK, Marwaha A, Pandey S. Use of radionuclide peritoneography in the diagnosis of pleuroperitoneal communication as a complication of continuous ambulatory peritoneal dialysis. Indian $J$ Nucl Med. 2005;20:4-8.

27. Rahman N, Lakhoo K. Patent processus vaginalis: a window to the abdomen. Afr J Paediatr Surg. 2009;6:116-117.

28. Mitoiu D, David C, Peride I, et al. Intra-peritoneal chronic loculation in peritoneal dialysis patients: a new medical management approach. Rom J Morphol Embryol. 2014;55:1409-1413.

29. Gudit S, Sudhakar P, Ram R, Prasad N, Prabhakar VV, Dakshinamurty KV. Peritoneal scintigraphy in the diagnosis of adhesions. Perit Dial Int. 2010;30:112-113.

30. Hou CH, Tsai TJ, Hsu KL. Peritoneopericardial communication after pericardiocentesis in a patient on continuous ambulatory peritoneal dialysis with dialysis pericarditis. Nephron. 1994;68:125-127.

31. Borzych D, Ley S, Schaefer F, et al. Dialysate leakage into pericardium in an infant on long-term peritoneal dialysis. Pediatr Nephrol. 2008;23:335-338.

32. Näther S, Anger H, Koall W, et al. Peritoneal leak and chronic pericardial effusion in a CAPD patient. Nephrol Dial Transplant. 1996;11:1155-1158.

33. Teoh CW, Nadel H, Armstrong K, Harris KC, White CT. Peritoneal-pericardial communication in an adolescent on peritoneal dialysis. Pediatr Nephrol. 2016;31: $153-156$. 